\title{
Impact of music on anxiety among patients undergoing eye surgery under topical anaesthesia
}

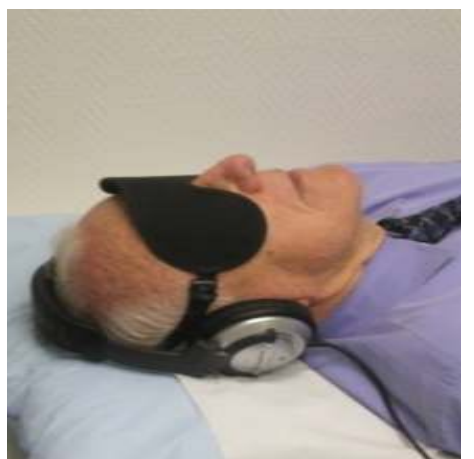

Gilles Guerrier*, David Boutboul, Sylvie Rondet, Dalila Hallal, Jacques Levy, Charles Marc Samama Hôpital Cochin, Assistance Publique-Hôpitaux de Paris, Université Paris Descartes, Paris, France *Corresponding author: gilles.guerrier@aphp.fr

Background and Goal of the Study: Awake ophthalmologic surgery is particularly stressful for patients. Music has long been known to reduce anxiety, minimize the need for sedatives, and make patients feel more at ease. The purpose of this pilot study was to evaluate the effect of music on anxiety in outpatients undergoing elective eye surgery under topical anaesthesia.

Materials and Methods: Sixty-two patients were prospectively and randomly assigned to hear relaxing music or no music via headphones just before surgery. Surgical Fear Questionnaire (SFQ) and Patient Satisfaction Questionnaire (PSQ) were used to assess anxiety before and after music session and overall patient satisfaction postoperatively. The proportion of patients receiving midazolam during surgery was also recorded. Comparisons were made using Chi-Square, or ANOVA, where appropriate.

\begin{tabular}{lccc} 
& $\begin{array}{c}\text { Music group } \\
\mathbf{n = 3 1}\end{array}$ & $\begin{array}{c}\text { No music group } \\
\mathbf{n}=\mathbf{3 1}\end{array}$ & p-value \\
\hline SFQ (mean 95\%Cl) & $23[0-45]$ & $65[10-88]$ & $<0.001$
\end{tabular}

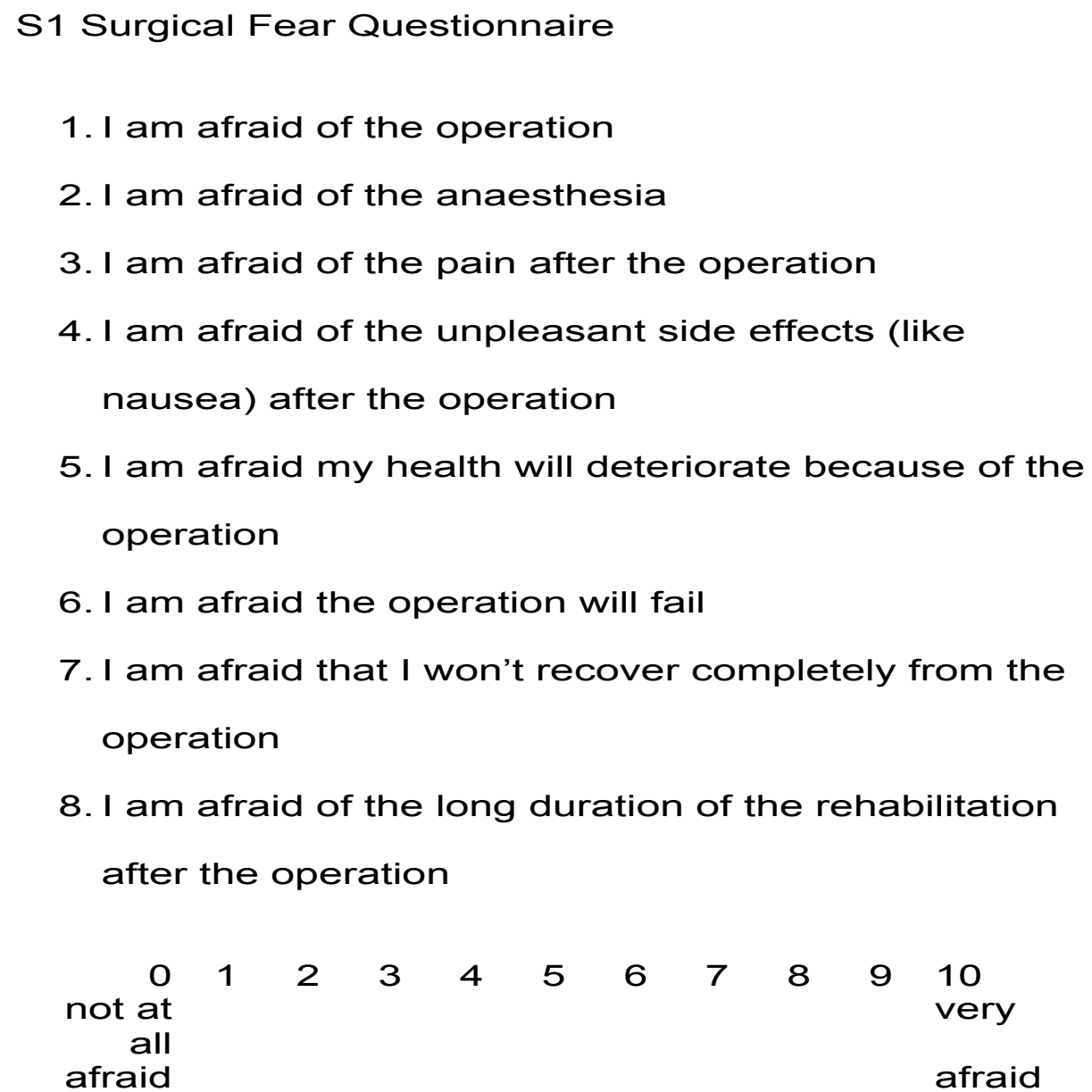
non-music group ( $16 \%$ vs $32 \%$; $=0.03)$. undergoing elective eye surgery under topical anaesthesia.

\section{Stimulating rhythm}

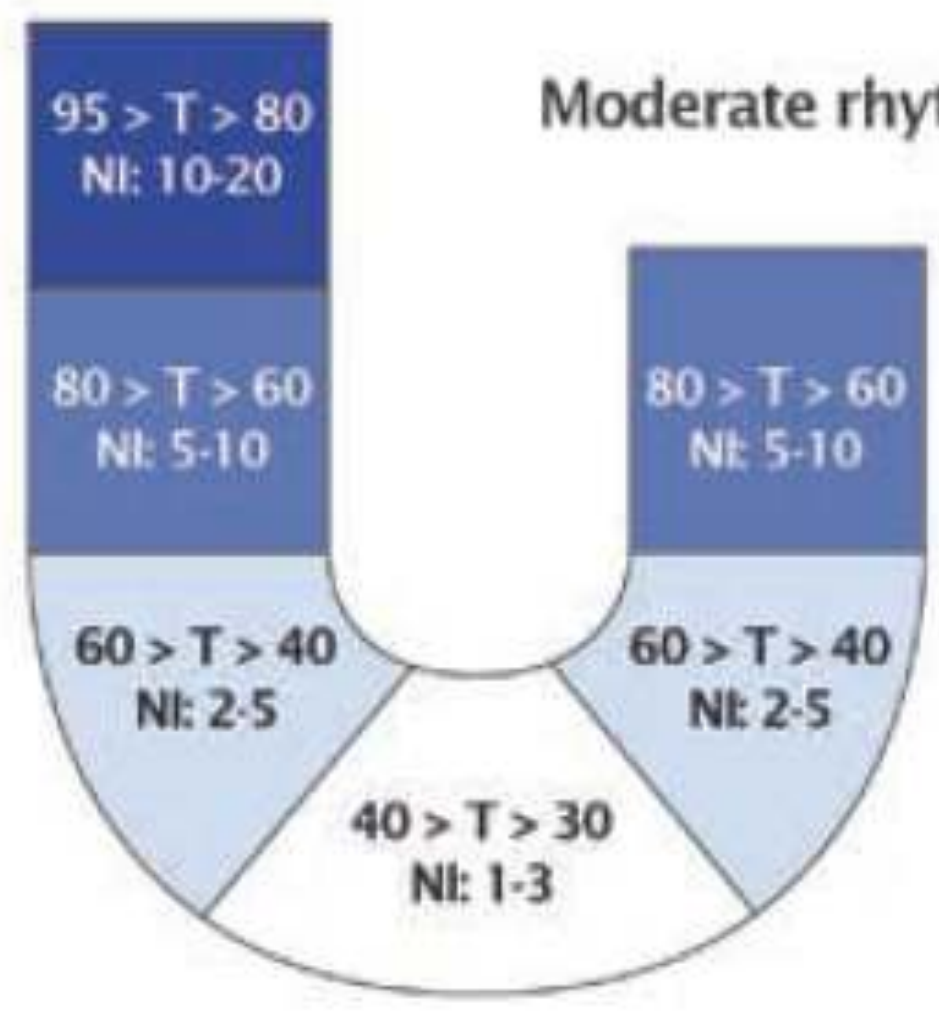

Slow rhythm (relaxation)

\section{0 minutes}

T: tempo (beats per minute) $\mathrm{Nl}$ : number of instruments

Results and Discussion: According to SFQ scores, anxiety was significantly reduced among the music group after listening to music (23 [0-45] vs 65 [10-88] $\mathrm{p}<0.001)$. Similarly, satisfaction scores were higher in the music group $(7 \mathrm{I}[43-80]$ vs $55[10-60] \mathrm{p}=0.02)$. The music group received significantly less sedatives during surgery compared with the

Conclusions: Music listening may be considered as an inexpensive, non-invasive, non-pharmacologic mean to reduce anxiety for patients

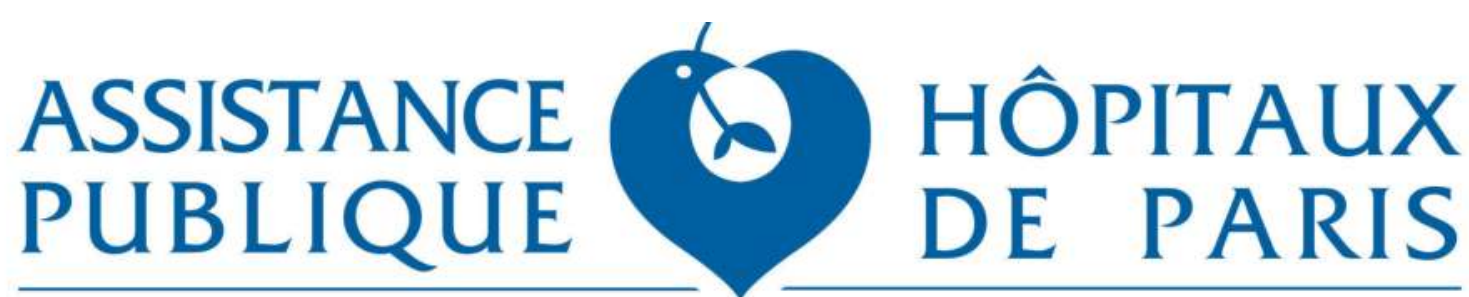

